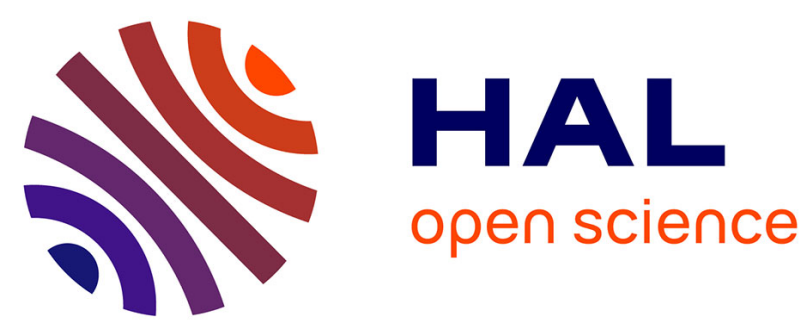

\title{
COL 3-04 - Abacavir/lamivudine/nevirapine comparée aux trithérapies classiques dans la cohorte Dat'AIDS : efficacité virologique similaire et meilleure tolérance à long terme
}

P. de Boissieu, M. Drame, F. Raffi, A. Cabié, I. Poizot-Martin, L. Cotte, P. Delobel, T. Huleux, D. Rey, F. Bani-Sadr

\section{To cite this version:}

P. de Boissieu, M. Drame, F. Raffi, A. Cabié, I. Poizot-Martin, et al.. COL 3-04 - Abacavir/lamivudine/nevirapine comparée aux trithérapies classiques dans la cohorte Dat'AIDS : efficacité virologique similaire et meilleure tolérance à long terme. 17es Journées Nationales d'Infectiologie, Jun 2016, Lille, France. inserm-01414591

HAL Id: inserm-01414591 https://www.hal.inserm.fr/inserm-01414591

Submitted on 12 Dec 2016

HAL is a multi-disciplinary open access archive for the deposit and dissemination of scientific research documents, whether they are published or not. The documents may come from teaching and research institutions in France or abroad, or from public or private research centers.
L'archive ouverte pluridisciplinaire HAL, est destinée au dépôt et à la diffusion de documents scientifiques de niveau recherche, publiés ou non, émanant des établissements d'enseignement et de recherche français ou étrangers, des laboratoires publics ou privés. 


\section{COL 3-03}

Tolérance de l'association fixe de tenofovir/emtricitabine/cobicistat/ elvitegravir utilisée en traitement après expositions à risque de transmission virale

E. Rouveix (1), S. Greffe (1), M. Chansombat (2), E. Reimann (1), L. Fonquernie (3), A. Simon (3), N. Valin (3), R. Tubiana (3), M-C. Meyohas (3), E. Bouvet (2)

(1) COREVIH Ile-de-France Ouest, Boulogne, France,

(2) COREVIH Ile-de-France Nord, Paris, France,

(3) COREVIH Ile-de-France Centre, Paris, France

Introduction Les recommandations nationales de Traitement Post Exposition (TPE) du groupe d'experts de 2013 préconisent l'utilisation de TDF/FTC associé à une IP/r. Les recommandations de traitement de l'infection VIH mises à jour en 2014 placent les anti-integrases en première intention au même titre que les anti-protéases. La formulation en STR (single tablet regimen) pourrait faciliter l'observance. Aussi, en raison du mode d'action, de la tolérance et de pharmacocinétique des antiintegrases, des données de la littérature concernant l'utilisation des antiintegrases en TPE et des recommandations américaines, l'utilisation du TDF/FTC/cobicistat/elvitegravir en TPE a été proposée dans 4 COREVIH. L'objectif de ce travail a été d'évaluer la tolérance du TDF/FTC/cobicistat/Elvitegravir dans cette indication et le nombre d'arrêts prématurés du traitement en lien avec des effets indésirables imputables au traitement.

Matériels et méthodes Tous les patients recevant du TDF/FTC/cobicistat/ elvitegravir en TPE dans les hôpitaux de 4 COREVIH ont été inclus, avec un recueil des EI imputables au TPE.

Résultats En 2015, 13 hôpitaux de 3 COREVIH ont traité 1566 expositions à risque viral (EAV): $1293(83 \%)$ sexuelles, $217(14 \%)$ professionnelles et 56 autres, concernant 1104 Hommes (70\%), âgés de 32 ans en moyenne. La sérologie VIH du patient source était inconnue dans $1171 / 1566$ cas $(81 \%)$, et $\mathrm{VIH}+$ dans 208 cas $(13 \%)$. Le TDF/FTC/cobicistat/elvitegravir était utilisé en TPE 844/1 566 fois (soit dans $62 \%$ des TPE), initié en CDAG (6\%), au SAU $(26 \%)$ ou en Service référent $(62 \%) ; 12 / 844$ patients (soit $1,4 \%$ ) ont dû arrêter le traitement en raison d'un EI (éruption cutanée : 3, troubles digestifs : 7, anomalies biologie hépatique : 2 , interaction médicamenteuse : 1). De plus, le recueil des effets indésirables n'ayant pas nécessité l'arrêt du TPE a été réalisé chez 603/844 patients : 150/603 (25\%) ont eu au moins un EI qui n'a pas nécessité de modifier le traitement et dont l'évolution a été favorable spontanément. Les EI ont été principalement digestifs (66\%), asthénie (21\%), neurologiques $(15 \%)$, anomalie biologique hépatique $(5 \%)$ ou rénale $(1 \%)$.

Conclusion Alors que dans les études historiques on note un taux d'arrêt du TPE de l'ordre de 10 à $22 \%$ lors de l'utilisation des molécules préconisées par le rapport d'expert (IP/r), le TDF/FTC/cobicistat/elvitegravir utilisé en TPE semble un traitement facile d'emploi $(1 \mathrm{cp} / \mathrm{j})$, bien toléré et responsable de peu d'arrêt prématuré, avec un taux même inférieur à celui du raltegravir (3 à $4 \%$ ) dans une utilisation similaire.

Liens d'intérêts déclarés : ER Voyages et accès aux congrès par GILEAD

\section{COL 3-04}

Abacavir/lamivudine/nevirapine comparée aux trithérapies classiques dans la cohorte Dat'AIDS : efficacité virologique similaire et meilleure tolérance à long terme

P. de Boissieu (1), M. Drame (1), F. Raffi (2), A. Cabié (3), I. Poizot-Martin (4), L. Cotte (5), P. Delobel (6), T. Huleux (7), D. Rey (8), F. Bani-Sadr (1)

(1) CHU Reims, Reims, France,

(2) CHU Nantes, Nantes, France,

(3) CHU Martinique, Fort de France, France,

(4) Hôpital Sainte-Marguerite, Marseille, France,

(5) Hospices Civils de Lyon, Lyon, France,

(6) CHU de Toulouse, Toulouse, France,

(7) Centre Hospitalier Tourcoing, Tourcoing, France,

(8) CHU Strasbourg, Strasbourg, France.

Introduction Les données sur l'efficacité virologique et la tolérance à longterme de l'association abacavir/lamivudine (ABC/3TC) et nevirapine (NVP) sont rares.
Matériels et méthodes Les taux d'arrêt du traitement antiretroviral $(\mathrm{ARV})$ pour échec virologique (EV) et/ou pour effets secondaires (ES) de l'association $\mathrm{ABC} / 3 \mathrm{TC} / \mathrm{NVP}$ ont été comparés à celles d' $\mathrm{ABC} / 3 \mathrm{TC}$ plus atazanavir boosté $(\mathrm{ATV} / \mathrm{r})$ ou darunavir $(\mathrm{DRV} / \mathrm{r})$, ou à celles de tenofovir/ emtricitabine (TDF/FTC) plus : ATV/r, ATV, DRV/r, efavirenz (EFV) ou NVP chez les patients recevant depuis plus de 6 mois le même traitement ARV, entre 2000 et 2013, dans la cohorte prospective, multicentrique DAT'AIDS.

Résultats Le suivi moyen des 15948 patients inclus dans l'étude était de $36 \pm 24$ mois. Les traitements ARV reçus étaient les suivants : $\mathrm{ABC} /$ $3 T C / N V P(n=1089)$, TDF/FTC/NVP $(\mathrm{n}=1542), \quad$ ABC/3TC/DRV/r $(\mathrm{n}=1065), \mathrm{ABC} / 3 \mathrm{TC} / \mathrm{ATV} / \mathrm{r}(\mathrm{n}=1847), \mathrm{TDF} / \mathrm{FTC} / \mathrm{ATV} / \mathrm{r}(\mathrm{n}=3519)$, $\mathrm{TDF} / \mathrm{FTC} / \mathrm{DRV} / \mathrm{r}(\mathrm{n}=2767), \mathrm{TDF} / \mathrm{FTC} / \mathrm{ATV}(\mathrm{n}=419)$ and TDF/FTC $/$ EFV $(n=3700)$. Les patients traités par $\mathrm{ABC} / 3 \mathrm{TC} / \mathrm{NVP}$ étaient naïfs d'ARV dans seulement $3 \%$ des cas. En analyse multivariée, le risque d'arrêt du traitement ARV pour EV après ajustement sur l'âge, le sexe, le mode de transmission du VIH, le taux de CD4 et le stade SIDA à l'initiation de chaque association d'ARV et le nombre de lignes d'ARV, était comparable entre 1 'ABC/3TC/NVP et les autres associations d'ARV, sauf pour TDF/FTC/ATV qui était associé à un risque plus élevé (HR : 1.99; 95\% CI : 1.29-3.06). L'association ABC/3TC/NVP était associée au risque le plus faible d'arrêt de traitement pour ES ; les autres associations d'ARV étant associées à un risque de 1.80 à 3,19 fois plus élevé $(\mathrm{p}<0.0001$ pour toutes les comparaisons).

Conclusion ABC/3TC/NVP prescrit en switch, possède une efficacité virologique à long-terme similaire et une tolérance à long-terme supérieure comparée aux trithérapies d'ARV les plus souvent prescrites.

Aucun lien d'intérêt

\section{COL 3-05}

Efficacité de la combinaison maraviroc-raltegravir chez des patients VIH-1 naïfs d'antirétroviraux : essai No Nuc-No Boost

L. Cotte (1), J. Durant (2), C. Brochier (1), J. Izopet (3), F. Raffi (4), M. Trabaud (1), F. Lucht (5), J. Cottalorda (2), J. Molina (6)

(1) Hospices Civils de Lyon, Lyon,

(2) CHU Nice, Nice,

(3) CHU Toulouse, Toulouse,

(4) CHU Nantes, Nantes,

(5) CHU St Etienne, St Etienne,

(6) Assistance Publique des Hôpitaux de Paris, Paris.

Introduction Les schémas thérapeutiques préférentiels chez les patients VIH naïs associent 2 inhibiteurs nucléos(t)idiques de la reverse transcriptase (INRT) et un antiprotéase boosté (IPb), un inhibiteur non-nucléosidique de la RT (INNRT) ou un anti-intégrase (II). Peu de données sont disponibles avec des combinaisons alternatives. La combinaison maraviroc (MRV) + Raltegravir (RAL) a montré des résultats décevants chez le patient pré-traité mais n'a jamais été étudiée chez le patient naïf. Nous rapportons les résultats de l'étude No Nuc-No Boost.

Matériels et méthodes Des patients infectés par VIH-1, naïs d'antirétroviraux ont été enrôlés dans une étude pilote. Les critères d'inclusion comportaient : CD4 $>200 / \mathrm{mm}^{3}$, charge virale VIH (CV) $10^{3}-10^{5} \mathrm{log}$ copies/mL, tropisme CCR5 (algorithme Geno2Pheno, FPR $20 \%$ ), absence de résistance aux INRT, INNRT, IPb, II. Tous les patients recevaient l'association MRV $300 \mathrm{mg}$ BID, RAL $400 \mathrm{mg}$ BID et tenofovir/emtricitabine (TDF/FTC) pendant 24 semaines. Les patients avec une CV $<50$ copies/mL à S20-22 arrêtaient TDF/FTC à S24 et poursuivaient MRV-RAL jusqu'à $S 48$. Le critère principal d'efficacité était la réponse virologique à $\mathrm{S} 48(\mathrm{CV}<50$ copies $/ \mathrm{mL})$.

Résultats Cinquante-cinq patients ont été présélectionnés, 34 recrutés et 1 patient a retiré son consentement, permettant l'analyse de 33 patients. Les raisons les plus fréquentes de non-inclusion étaient un virus non-R5 $(\mathrm{n}=13)$ et/ou une $\mathrm{CV}$ en dehors des limites $(\mathrm{n}=4)$. La plupart des patients étaient des hommes $(94 \%)$, caucasiens $(91 \%)$, homosexuels (82\%), d'âge médian 42 ans. La médiane des CD4 à l'inclusion était de $453 / \mathrm{mm}^{3}$ et la CV de $4,3 \log$ copies $/ \mathrm{mL} .36 \%$ des patients étaient infectés par un sous-type non-B. Tous les génotropismes R5 étaient confirmés par phénotropisme. Un patient avec une $\mathrm{CV}>50$ copies $/ \mathrm{mL}$ à $\mathrm{S} 24$ n'est pas entré dans la phase de simplification. Trois patients présentaient un échec virologique à respectivement $\mathrm{S} 36, \mathrm{~S} 44$ et $\mathrm{S} 48$. Le taux de succès virologique était de $97 \%$ à $\mathrm{S} 24$ et de $88 \% / 91 \%$ (ITT/per protocole) à S48. La 